\title{
On topological homotopy groups and relation to Hawaiian groups
}

\author{
Ameneh Babaee (D), Behrooz Mashayekhy (D), Hanieh Mirebrahimi* (D), Hamid \\ Torabi (D), Mahdi Abdullahi Rashid (D), Seyyed Zeynal Pashaei (iD \\ Department of Pure Mathematics, Center of Excellence in Analysis on Algebraic Structures, Ferdowsi \\ University of Mashhad, P.O.Box 1159-91775, Mashhad, Iran
}

\begin{abstract}
By generalizing the whisker topology on the $n$th homotopy group of pointed space $\left(X, x_{0}\right)$, denoted by $\pi_{n}^{w h}\left(X, x_{0}\right)$, we show that $\pi_{n}^{w h}\left(X, x_{0}\right)$ is a topological group if $n \geq 2$. Also, we present some necessary and sufficient conditions for $\pi_{n}^{w h}\left(X, x_{0}\right)$ to be discrete, Hausdorff and indiscrete. Then we prove that $L_{n}\left(X, x_{0}\right)$ the natural epimorphic image of the Hawaiian group $\mathcal{H}_{n}\left(X, x_{0}\right)$ is equal to the set of all classes of convergent sequences to the identity in $\pi_{n}^{w h}\left(X, x_{0}\right)$. As a consequence, we show that $L_{n}\left(X, x_{0}\right) \cong L_{n}\left(Y, y_{0}\right)$ if $\pi_{n}^{w h}\left(X, x_{0}\right) \cong \pi_{n}^{w h}\left(Y, y_{0}\right)$, but the converse does not hold in general, except for some conditions. Also, we show that on some classes of spaces such as semilocally $n$-simply connected spaces and $n$-Hawaiian like spaces, the whisker topology and the topology induced by the compact-open topology of $n$-loop space coincide. Finally, we show that $n$-SLT paths can transfer $\pi_{n}^{w h}$ and hence $L_{n}$ isomorphically along its points.
\end{abstract}

Mathematics Subject Classification (2010). 55Q05, 55Q20, 55P65, 55Q52

Keywords. Whisker topology, Hawaiian group, $n$-dimensional Hawaiian earring, harmonic archipelago

\section{Introduction and motivation}

E.H. Spanier introduced a topology on the fundamental group [21, Theorem 13], named whisker topology by N. Brodskiy et al. [6]. It is originally defined on a quotient of the path space introduced in [6, Definition 4.2] including the fundamental group as a fibre. It was shown that for a pointed space $\left(X, x_{0}\right)$ the restriction of the whisker topology on $\pi_{1}\left(X, x_{0}\right)$ is generated by the basis $\bigcup_{[\alpha] \in \pi_{1}\left(X, x_{0}\right)}\left\{[\alpha] \pi_{1}(i) \pi_{1}\left(U, x_{0}\right) \mid U\right.$ is an open neighbourhood of $x_{0}$ and $i: U \rightarrow X$ is the inclusion map $\}$.

Another topology on the fundamental group was defined in [5], called lasso topology. In general, the lasso topology makes the fundamental group a topological group, but not the whisker topology. As an example, if $\mathbb{H} \mathbb{E}^{1}$ denotes the 1-dimensional Hawaiian earring, the inverse operation of $\pi_{1}^{w h}\left(\mathbb{H E}^{1}, \theta\right)$ is not continuous [5]. Also, if $\pi_{1}^{q t o p}$ denotes the

\footnotetext{
*Corresponding Author.

Email addresses: am.babaee@mail.um.ac.ir (A. Babaee), bmashf@um.ac.ir (B. Mashayekhy), h_mirebrahimi@um.ac.ir (H. Mirebrahimi), h.torabi@um.ac.ir (H. Torabi), mbinev@mail.um.ac.ir (M. Abdullahi Rashid), Pashaei.seyyedzeynal@stu.um.ac.ir (S.Z. Pashaei)

Received: 14.05.2019; Accepted: 30.10.2019
} 
fundamental group equipped with the topology induced by compact-open topology of 1loop space, then the multiplication of $\pi_{1}^{q t o p}\left(\mathbb{H} \mathbb{E}^{1}\right)$ is not continuous [10]. This topology was generalized to higher dimension by F.H. Ghane et al. [14] induced by the compact-open topology of $n$-loop space.

In Section 2, we generalize the whisker topology to the $n$th homotopy group, $n \in \mathbb{N}$, denoted by $\pi_{n}^{w h}\left(X, x_{0}\right)$, using subgroup topology which makes $\pi_{n}\left(X, x_{0}\right)$ a left topological group for any pointed space $\left(X, x_{0}\right)$. We show that for $n \geq 2$, the whisker topology makes $\pi_{n}\left(X, x_{0}\right)$ a topological group.

In Section 3, we establish some necessary and sufficient conditions for $\pi_{n}^{w h}\left(X, x_{0}\right)$ to be discrete, Hausdorff, and indiscrete. For instance, an equivalent condition for $\pi_{n}^{w h}\left(X, x_{0}\right)$ to be discrete, is semi-locally $n$-simply connectedness at $x_{0}$. Also, we show that any subgroup $H \leqslant \pi_{n}^{w h}\left(X, x_{0}\right)$ is closed if and only if $X$ is $n$-homotopically Hausdorff relative to $H$ at $x_{0}$.

It is well-known that a path induces an isomorphism on homotopy groups at its beginning and end points. But this isomorphism is not necessarily continuous. Brodskiy et al. [6, Proposition 4.10] showed that the 1-dimensional Hawaiian earring is a path connected space with non-homeomorphic fundamental groups equipped with the whisker topology at some different points. Moreover, they defined a kind of path, called an SLT-path, which makes the induced isomorphism on fundamental groups continuous. We generalize SLTpaths to $n$-SLT paths in order to induce continuous isomorphism on the $n$th homotopy groups.

Section 4 discusses the relation between topological homotopy groups and Hawaiian groups. For $n \geq 1$, the $n$th Hawaiian group was defined as a functor from $h T o p_{*}$, the pointed homotopy category, to Groups, the category of groups (see [17]). Assume that $\mathbb{H} \mathbb{E}^{n}=\bigcup_{k \in \mathbb{N}} \mathbb{S}_{k}^{n}$ denotes the $n$-dimensional Hawaiian earring introduced in [9], where $\mathbb{S}_{k}^{n}$ is the $n$-sphere with radius $1 / k$ centered at $(1 / k, 0, \ldots, 0)$ in $\mathbb{R}^{n+1}$, and $\theta$ denotes the origin.

Definition $1.1([17])$. Let $\left(X, x_{0}\right)$ be a pointed space, and let $[\cdot]$ denote the class of pointed homotopy. The $n$th Hawaiian group of $\left(X, x_{0}\right)$, is defined by $\mathcal{H}_{n}\left(X, x_{0}\right)=\{[f]$ : $\left.f:\left(\mathbb{H E}^{n}, \theta\right) \rightarrow\left(X, x_{0}\right)\right\}$. For any $[f],[g] \in \mathcal{H}_{n}\left(X, x_{0}\right)$, multiplication is induced by $\left.(f * g)\right|_{\mathbb{S}_{k}^{n}}=\left.\left.f\right|_{\mathbb{S}_{k}^{n}} * g\right|_{\mathbb{S}_{k}^{n}}(k \in \mathbb{N})$.

The operation of the $n$th Hawaiian group implies that for all $n \in \mathbb{N}$, the following map

$$
\varphi: \mathcal{H}_{n}\left(X, x_{0}\right) \rightarrow \prod_{\aleph_{0}} \pi_{n}\left(X, x_{0}\right),
$$

defined by $\varphi([f])=\left(\left[\left.f\right|_{\mathbb{S}_{1}^{n}}\right],\left[\left.f\right|_{\mathbb{S}_{2}^{n}}\right], \ldots\right)$ is a homomorphism. For every pointed space $\left(X, x_{0}\right)$, homomorphic image $\operatorname{im}(\varphi)$ denoted by $L_{n}\left(X, x_{0}\right)$ which is equal to a special subset of $\prod_{\aleph_{0}} \pi_{n}\left(X, x_{0}\right)[3$, Definition 2.6] as follows.

Definition $1.2([3])$. Let $\left(X, x_{0}\right)$ be a pointed space and $n \geq 1$. Then $L_{n}\left(X, x_{0}\right)$ is the subset of $\prod_{\aleph_{0}} \pi_{n}\left(X, x_{0}\right)$ consisting of all sequences of homotopy classes $\left\{\left[f_{k}\right]\right\}$, whenever $i m\left(f_{k}\right) \subseteq U$ for all $k \in \mathbb{N}$ except a finite number, if $U$ is an open set containing $x_{0}$.

For instance, if $X$ is a metric space, then $L_{n}\left(X, x_{0}\right)$ is the subset of $\prod_{\aleph_{0}} \pi_{n}\left(X, x_{0}\right)$ consisting of all classes of uniform convergent sequences to the constant map at $x_{0}$.

It was proved that $L_{n}\left(X, x_{0}\right)=\varphi\left(\mathcal{H}_{n}\left(X, x_{0}\right)\right)$, and hence it is a subgroup of $\prod_{\aleph_{0}} \pi_{n}\left(X, x_{0}\right)$ (see [3, Theorem 2.7]). Therefore, one can consider the homomorphism $\varphi$ as an epimorphism from $\mathcal{H}_{n}\left(X, x_{0}\right)$ onto $L_{n}\left(X, x_{0}\right)$.

In Section 4 , we attend the relation of $L_{n}\left(X, x_{0}\right)$ and $\pi_{n}^{w h}\left(X, x_{0}\right)$, for any pointed space $\left(X, x_{0}\right)$, and we see that they are closely dependent on each other. In fact, it is shown that $L_{n}\left(X, x_{0}\right)$ is equal to the set of all convergent sequences to the identity in $\pi_{n}^{w h}\left(X, x_{0}\right)$. As a consequence, we see that on $n$-Hawaiian like spaces, the two topologies of $\pi_{n}^{w h}$ and $\pi_{n}^{q t o p}$ coincide. Then, we prove that $L_{n}\left(X, x_{0}\right) \cong L_{n}\left(Y, y_{0}\right)$, whenever $\pi_{n}^{w h}\left(X, x_{0}\right) \cong \pi_{n}^{w h}\left(Y, y_{0}\right)$ 
as left topological groups, for any pointed spaces $\left(X, x_{0}\right)$ and $\left(Y, y_{0}\right)$. It implies a sufficient condition to fix the structure of $L_{n}$ at different points which is the existence of some two sided small $n$-loop transfer ( $n$-SLT) path. Finally, we study two groups $L_{1}(\mathbb{H} \mathbb{A}, a)$ and $L_{1}(\mathbb{H} \mathbb{A}, \theta)$, where $\mathbb{H} \mathbb{A}$ is the harmonic archipelago, $\theta$ is the origin, and $a$ is another point. We prove that $L_{1}(\mathbb{H} \mathbb{A}, a) \nRightarrow L_{1}(\mathbb{H} \mathbb{A}, \theta)$ to see that the existence of $n$-SLT paths is necessary to induce isomorphism on $L_{n}$ and topological homotopy groups at different points.

Throughout this article all homotopies are relative to the base point.

\section{Whisker topology on homotopy groups}

In this section, we intend to introduce the whisker topology on the $n$th homotopy groups. The whisker topology on the fundamental group has been introduced and discussed by Brodskiy et al. in [6].

Let $\left(X, x_{0}\right)$ be a pointed space, and let $n \geq 1$. For each open neighbourhood $U$ of $x_{0}$ in $X$, the inclusion map $i: U \rightarrow X$ induces the natural homomorphism $\pi_{n}(i): \pi_{n}\left(U, x_{0}\right) \rightarrow$ $\pi_{n}\left(X, x_{0}\right)$. Hence, $\pi_{n}(i)\left(\pi_{n}\left(U, x_{0}\right)\right)$ is a subgroup of $\pi_{n}\left(X, x_{0}\right)$. Also, for any open neighbourhoods $U$ and $V$ containing $x_{0}$, we have

$$
\pi_{n}\left(i_{1}\right)\left(\pi_{n}\left(U \cap V, x_{0}\right)\right) \leq \pi_{n}\left(i_{2}\right)\left(\pi_{n}\left(U, x_{0}\right)\right) \cap \pi_{n}\left(i_{3}\right)\left(\pi_{n}\left(V, x_{0}\right)\right),
$$

where $i_{1}, i_{2}$, and $i_{3}$ are corresponding inclusion maps. Therefore, the collection of all such subgroups forms a neighbourhood family on $\pi_{n}\left(X, x_{0}\right)$ which is defined as follows.

Definition 2.1 ([4]). Let $G$ be a group with the identity element $e$. A nonempty family $\Sigma$ of subgroups of $G$ is called a neighbourhood family whenever for any $S, S^{\prime} \in \Sigma$, there exists $S^{\prime \prime} \in \Sigma$, such that $S^{\prime \prime} \subseteq S \cap S^{\prime}$. Let $g \in G$ and $\Sigma$ be a neighbourhood family, then the set of all cosets $\{g S: S \in \Sigma\}$ forms a local basis at $g$. Thus, the set $\{g S: g \in G, S \in \Sigma\}$ is a basis for a topology on $G$ which is called a subgroup topology. The intersection $S_{\Sigma}=\bigcap_{S \in \Sigma} S$ is called the infinitesimal subgroup for the neighbourhood family $\Sigma$.

Using the above definition, we are going to endow the $n$th homotopy group with a topology called whisker topology. The whisker topology on the fundamental group has been defined as a subspace of a path space introduced in [6]. Note that one can consider the fundamental group as the 1st homotopy group.

Definition 2.2. Let $\left(X, x_{0}\right)$ be a pointed space, and $n \geq 1$. By Inequality (2.1),

$$
\Sigma=\left\{\pi_{n}(i) \pi_{n}\left(U, x_{0}\right) \mid \mathrm{U} \text { is an open subset of } X \text { containing } x_{0}\right\},
$$

is a neighbourhood family on $\pi_{n}\left(X, x_{0}\right)$. The whisker topology on the $n$th homotopy group, $\pi_{n}\left(X, x_{0}\right)$, of a pointed topological space $\left(X, x_{0}\right)$ is the subgroup topology determined by the neighbourhood family $\Sigma$ which is denoted by $\pi_{n}^{w h}\left(X, x_{0}\right)$.

Note that for any $n$-loop $\alpha$ the collection $\Sigma_{[\alpha]}=\left\{[\alpha] \pi_{n}(i) \pi_{n}\left(U, x_{0}\right) \mid U\right.$ is an open subset of $X$ containing $\left.x_{0}\right\}$ is a local basis at $[\alpha] \in \pi_{n}^{w h}\left(X, x_{0}\right)$. Then we have the following result.

Lemma 2.3. Let $\left(X, x_{0}\right)$ be a pointed space, and let $n \geq 1$. If $X$ has a countable local basis at $x_{0}$, then $\pi_{n}^{w h}\left(X, x_{0}\right)$ is first countable.

Let $n \geq 1$. Recall that an $n$-loop $\alpha:\left(\mathbb{S}^{n}, 1\right) \rightarrow\left(X, x_{0}\right)$ is said to be small if it has a homotopic equivalent in every open neighbourhood of $x_{0}$ [20], and $\pi_{n}^{s}\left(X, x_{0}\right)$ denotes the collection of all classes of small $n$-loops at $x_{0}$. Let $[\alpha] \in \bigcap\left\{\pi_{n}(i) \pi_{n}\left(U, x_{0}\right) \mid U\right.$ is an open neighbourhood of $x_{0}$, then $\alpha$ has a homotopic representative in any open neighbourhood of $x_{0}$, that is, $\alpha$ is a small $n$-loop at $x_{0}$. Thus, the infinitesimal subgroup of $\pi_{n}^{w h}\left(X, x_{0}\right)$ is equal to $\pi_{n}^{s}\left(X, x_{0}\right)$. It is easy to see that $\pi_{n}^{w h}\left(X, x_{0}\right)$ is indiscrete if and only if $\pi_{n}^{s}\left(X, x_{0}\right)=\pi_{n}\left(X, x_{0}\right)$. As an example, if $\mathbb{H} \mathbb{A}$ denotes the harmonic archipelago space, and $\theta$ denotes the origin, then $\pi_{1}^{w h}(\mathbb{H} \mathbb{A}, \theta)$ is indiscrete. Moreover, if $\pi_{n}^{w h}\left(X, x_{0}\right)$ is discrete, then $\pi_{n}^{s}\left(X, x_{0}\right)$ is the trivial subgroup. The converse does not hold, in general. As a 
counterexample, for the $n$-dimensional Hawaiian earring, $\mathbb{H} \mathbb{E}^{n}$ at the origin $\theta, \pi_{n}^{s}\left(\mathbb{H}^{n}, \theta\right)$ is trivial, but $\pi_{n}^{w h}\left(\mathbb{H}_{\mathbb{E}}^{n}, \theta\right)$ is not discrete (see Example 4.6).

Remark 2.4 ([4]). With the previous assumption and notation, for $g \in G$ and $S \in \Sigma$, a basic set $g S$ is both open and closed in the subgroup topology, since the cosets of a given subgroup form a partition of $G$. The subgroup topology is a homogeneous space, since left translations by elements of $G$ determine self-homeomorphisms on $G$. However, the group $G$ is not necessarily a topological group, since the right translation by a fixed element of $G$ is not continuous, in general. The infinitesimal subgroup is a closed subgroup in the subgroup topology on $G$ induced by $\Sigma$. Indeed, $S_{\Sigma}$ is the closure of the identity $e \in G$, and its coset $g S_{\Sigma}$ is the closure of the element $g \in G$.

Note that $\pi_{n}^{s}\left(X, x_{0}\right)$ is a closed subgroup of $\pi_{n}^{w h}\left(X, x_{0}\right)$, but it may not be open, in general. However, some nice properties occur if it is open. The following proposition generalizes Proposition 2.4 in [1], by a similar argument, for the whisker topology on the $n$th homotopy group $(n \geq 1)$.

Proposition 2.5. Let $\left(X, x_{0}\right)$ be a pointed topological space, then the following statements are equivalent.

(1) $\pi_{n}^{s}\left(X, x_{0}\right)$ is an open subgroup of $\pi_{n}^{w h}\left(X, x_{0}\right)$.

(2) Every closed subgroup of $\pi_{n}^{w h}\left(X, x_{0}\right)$ is an open subgroup.

(3) A subgroup $H$ of $\pi_{n}^{w h}\left(X, x_{0}\right)$ is open if and only if it is closed.

(4) A subgroup $H$ of $\pi_{n}^{w h}\left(X, x_{0}\right)$ is open if and only if $\pi_{n}^{s}\left(X, x_{0}\right) \leq H$.

By Remark 2.4, every subgroup topology on a given group makes it a homogeneous space, and hence, it is a left topological group. It was shown in [1, Proposition 2.1] that if a subgroup topology on a group makes it a right topological group, then it is a topological group. Since $\pi_{n}\left(X, x_{0}\right)$ is abelian, for $n \geq 2$, the right translation map $r_{\alpha}: \pi_{n}\left(X, x_{0}\right) \rightarrow \pi_{n}\left(X, x_{0}\right)$ by the rule $r_{\alpha}([\beta])=[\alpha * \beta]$ is equal to the left translation map $l_{\alpha}: \pi_{n}\left(X, x_{0}\right) \rightarrow \pi_{n}\left(X, x_{0}\right)$ by the rule $l_{\alpha}([\beta])=[\beta * \alpha]$, for any $[\alpha] \in \pi_{n}\left(X, x_{0}\right)$. Hence, $r_{\alpha}$ is continuous for all $[\alpha] \in \pi_{n}\left(X, x_{0}\right)$. Therefore, $\pi_{n}^{w h}\left(X, x_{0}\right)$ is a right topological group, too, for $n \geq 2$. As a consequence we have the following result.

Proposition 2.6. Let $\left(X, x_{0}\right)$ be a pointed space. If $n \geq 2$, then $\pi_{n}^{w h}\left(X, x_{0}\right)$ is a topological group.

Since $\pi_{1}\left(X, x_{0}\right)$ is not necessarily an abelian group, Proposition 2.6 does not hold in the case of $n=1$. As an example $\pi_{1}^{w h}\left(\mathbb{H} \mathbb{E}^{1}, \theta\right)$ is not a topological group [5]. For $n=1$, there exists a necessary and sufficient condition called SLTL, established in [16, Proposition 2] for $\pi_{1}^{w h}\left(X, x_{0}\right)$ to be a topological group.

Fisher et al. [11, Theorem 4.10 (d)] proved that if $X$ is metric, then so is the path space $\widetilde{X}$, whenever $X$ is shape injective. Also, by Lemma 2.3, if $X$ has a countable local basis at $x_{0}$, then $\pi_{n}^{w h}\left(X, x_{0}\right)$ is first countable. In the following, we see that for $n \geq 2$, there is sufficient conditions for $\pi_{n}^{w h}\left(X, x_{0}\right)$ to be metric.

G.R. Conner et al. [7] defined the homotopically Hausdorff property. This property has been extended to $n$-homotopically Hausdorff property by H. Passandideh et al. [20, Definition 3.3] for $n \geq 1$. A space $X$ is called $n$-homotopically Hausdorff at $x_{0}$ whenever for each essential $n$-loop $\alpha$ in $X$ at $x_{0}$, there exists an open neighbourhood $U$ of $x_{0}$, containing no $n$-loop at $x_{0}$ homotopic to $\alpha$, that is $\pi_{n}^{s}\left(X, x_{0}\right)=\langle e\rangle$.

Corollary 2.7. Let $X$ be a space having a countable local basis at $x_{0}$, and let $n \geq 2$. If $X$ is n-homotopically Hausdorff at $x_{0}$, then $\pi_{n}^{w h}\left(X, x_{0}\right)$ is a metric topological group.

Proof. By Proposition 2.6, $\pi_{n}^{w h}\left(X, x_{0}\right)$ is a topological group. If $X$ is $n$-homotopically Hausdorff at $x_{0}$, then by [4, Theorem $\left.2.9(\mathrm{c})\right], \pi_{n}^{w h}\left(X, x_{0}\right)$ is Hausdorff and thus, satisfies $T_{1}$-separation axiom. Hence, by $\left[2\right.$, Theorem 3.3 .12$, p. 155], $\pi_{n}^{w h}\left(X, x_{0}\right)$ is metric if and 
only if it is first countable. Since $X$ has a countable local basis at $x_{0}$, by Lemma 2.3, $\pi_{n}^{w h}\left(X, x_{0}\right)$ is first countable. Therefore, $\pi_{n}^{w h}\left(X, x_{0}\right)$ is a metric topological group.

Note that Ghane et al. in [14, Page 264] by a filter base which forms a fundamental system of neighborhoods of the identity element gave a topology to the homotopy group $\pi_{n}\left(X, x_{*}\right)$ denoted by $\pi_{n}^{l i m}\left(X, x_{*}\right)$. It should be mentioned that one can prove the topology of $\pi_{n}^{\lim }\left(X, x_{*}\right)$ coincides with the whisker topology $\pi_{n}^{w h}\left(X, x_{*}\right)$.

\section{Whisker topology and local properties}

In this section, we are going to find some relationships between topological properties of $\pi_{n}^{w h}\left(X, x_{0}\right)$ and local properties of the space $X$ at the base point $x_{0}$. Moreover, we discuss conditions for $\pi_{n}^{w h}\left(X, x_{0}\right)$ to be invariant with respect to the base point $x_{0}$.

The following proposition states the equivalence condition for $\pi_{n}^{w h}\left(X, x_{0}\right)$ to be discrete. Recall from [14, Definition 3.1] that a pointed topological space $\left(X, x_{0}\right)$ is called semilocally $n$-simply connected at $x_{0}$ if there exists an open neighbourhood $U$ at $x_{0}$ for which any $n$-loop in $U$ based at $x_{0}$ is nulhomotopic in $X$.

Proposition 3.1. Let $\left(X, x_{0}\right)$ be a pointed space, and let $n \geq 1$. Then $\pi_{n}^{w h}\left(X, x_{0}\right)$ is discrete if and only if $X$ is semilocally $n$-simply connected at $x_{0}$.

Proof. If $X$ is semilocally $n$-simply connected at $x_{0}$, then there is an open neighbourhood $U$ of $x_{0}$ such that $\pi_{n}(i) \pi_{n}\left(U, x_{0}\right)$ is trivial. Since $\pi_{n}(i) \pi_{n}\left(U, x_{0}\right) \in \Sigma$, then $\pi_{n}^{w h}\left(X, x_{0}\right)$ is discrete. Conversely, if $\pi_{n}^{w h}\left(X, x_{0}\right)$ is discrete, then the trivial subgroup is open in $\pi_{n}^{w h}\left(X, x_{0}\right)$. Since $\Sigma$ is a local basis, there is an open neighbourhood $U$ of $x_{0}$, such that $\pi_{n}(i) \pi_{n}\left(U, x_{0}\right) \subseteq\{e\}$, that is $\pi_{n}(i) \pi_{n}\left(U, x_{0}\right)=\{e\}$. Hence $X$ is semi-locally $n$-simply connected at $x_{0}$.

H. Fischer et al. [11, ] defined homotopically Hausdorff property relative to $H$, where $H$ is a subgroup of $\pi_{1}\left(X, x_{0}\right)$. Brodskiy et al. [5, Definition 4.11] generalized this concept to $(G, H)$-homotopically Hausdorff property, where $H \leqslant G \leqslant \pi_{1}\left(X, x_{0}\right)$. A space $X$ is called $(G, H)$-homotopically Hausdorff, if for any $g \in G-H$ and any path $\alpha$ originating at $x_{0}$, there is an open neighbourhood $U$ of $\alpha(1)$ in $X$ such that none of the elements of $H g$ can be expressed as $\left[\alpha * \gamma * \alpha^{-1}\right]$ for any loop $\gamma$ in $(U, \alpha(1))$. In the following, we define $n$-homotopically Hausdorff property relative to a pair of subgroups $(G, H)$ at the base point $x_{0}$, where $H \leqslant G \leqslant \pi_{n}\left(X, x_{0}\right)(n \geq 1)$.

Definition 3.2. Let $H \leqslant G \leqslant \pi_{n}\left(X, x_{0}\right)$, and let $n \geq 1$. We say that $X$ is $n$-homotopically Hausdorff relative to $(G, H)$ at $x_{0}$, if for each $g \in G-H$, there exists an open neighbourhood $U$ of $x_{0}$, such that no element of $H g$ can be expressed as $[\gamma]$, for any $n$-loop $\gamma$ in $\left(U, x_{0}\right)$.

Note that $X$ is $n$-homotopically Hausdorff relative to $G$, if $X$ is $n$-homotopically Hausdorff relative to $(G,\{e\})$ at $x_{0}$. Although, $n$-homotopically Hausdorff property relative to $(G, H)$ at $x_{0}$ is defined closely to $(G, H)$ - homotopically Hausdorff property [5, Definition 4.11], if $X$ is 1-homotopically Hausdorff relative to $(G, H), H \leqslant G \leqslant \pi_{1}\left(X, x_{0}\right)$ at any point in the sense of Definition 3.2, it does not need to be $(G, H)$-homotopically Hausdorff in the sense of [5].

It is proved that $X$ is homotopically Hausdorff relative to $(G, H)$, if $H$ is closed in $G$ endowed with a new topology [5, Lemma 4.14] called lasso topology in [6]. Also, Fisher et al. [11, ] proved that homotopically Hausdorff relative to a subgroup $H$ is equivalent to Hausdorffness of a path space equipped with a suitable topology. The following theorem presents a similar explanation of [5, Proposition 4.12 and Lemma 4.16], [11, Lemma 2.10 and Proposition 6.3]. 
Theorem 3.3. Let $\left(X, x_{0}\right)$ be a pointed space, $H \leqslant G \leqslant \pi_{n}^{w h}\left(X, x_{0}\right)$, and $n \geq 1$. Then the following statements are equivalent.

(i) (i) $X$ is n-homotopically Hausdorff relative to $(G, H)$ at $x_{0}$.

(ii) $H$ is a closed subgroup of $G$.

(iii) The coset space $\frac{G}{H}$, with the quotient topology, is a homogenous Hausdorff space.

Proof. (1) $((i) \Rightarrow(i i))$ Let $X$ be $n$-homotopically Hausdorff relative to $(G, H)$ at $x_{0}$. Then, for every $g \in G-H$, there exists an open neighbourhood $U_{g}$ of $x_{0}$, such that $\pi_{n}(i) \pi_{n}\left(U_{g}, x_{0}\right) \cap H g=\emptyset$, where $i: U \hookrightarrow X$ is the inclusion map. Assume that $g \in G-H$ and $g \in \bar{H}$. Thus, for each open neighbourhood $V$ of $g$ in $G, V \cap H \neq \emptyset$. Put $V=g \pi_{n}(i) \pi_{n}\left(U_{g}, x_{0}\right) \cap G$. Then $\left(g \pi_{n}(i) \pi_{n}\left(U_{g}, x_{0}\right) \cap G\right) \cap H \neq \emptyset$. Since $H \leqslant G$, $g \pi_{n}(i) \pi_{n}\left(U_{g}, x_{0}\right) \cap G \cap H=g \pi_{n}(i) \pi_{n}\left(U_{g}, x_{0}\right) \cap H$. Let $h \in g \pi_{n}(i) \pi_{n}\left(U_{g}, x_{0}\right) \cap H$. Then $g^{-1} h \in \pi_{n}(i) \pi_{n}\left(U_{g}, x_{0}\right)$. Since $\pi_{n}(i) \pi_{n}\left(U_{g}, x_{0}\right)$ is a subgroup of $\pi_{n}\left(X, x_{0}\right)$, $h^{-1} g \in \pi_{n}(i) \pi_{n}\left(U_{g}, x_{0}\right)$. Since $H$ is a subgroup of $\pi_{n}\left(X, x_{0}\right), h^{-1} \in H$, and so $h^{-1} g \in H g$. But we showed that $h^{-1} g$ is an element of $\pi_{n}(i) \pi_{n}\left(U_{g}, x_{0}\right) \cap H g$ which is a contradiction to $\pi_{n}(i) \pi_{n}\left(U_{g}, x_{0}\right) \cap H g=\emptyset$. Therefore, if $g \in \bar{H}$, then $g \notin G-H$, that is $H$ is closed in $G$.

(2) $((i i) \Rightarrow($ iii $))$ Let $H$ be closed in $G$. Since $\pi_{n}^{w h}\left(X, x_{0}\right)$ is a left topological group, its subgroup $G$ is also a left topological group. Thus, by [2, Theorem 1.5.1, p. 37], the coset space $\frac{G}{H}$ endowed with the quotient topology is a homogeneous $T_{1}$-space. Since each $T_{1}$-space is a $T_{0}$-space, the coset space $\frac{G}{H}$ is a $T_{0}$-space. By $[4$, Theorem 3.4 , p. 19], part $((i i i) \Rightarrow(i))$, the coset space $\frac{G}{H}$ is Hausdorff.

(3) $($ iii $) \Rightarrow(i))$ Let the coset space $\frac{G}{H}$ be Hausdorff. Then, for each $g \in G-H$, there exist open neighbourhoods $V$ and $W$ of $H$ and $H g$, respectively, in $\frac{G}{H}$, such that $H \in V$ and $H g \in W$, and $V \cap W=\emptyset$. Thus, $H g \notin V$, or equivalently, there is no $h \in H$ such that $h g \in q^{-1}(V)$, that is $H g \cap q^{-1}(V)=\emptyset$, where $q: G \rightarrow \frac{G}{H}$ is the quotient map. Since $V$ is an open neighbourhood of $H$ in $\frac{G}{H}$, and $q$ is continuous, $q^{-1}(V)$ is an open neighbourhood of the identity in $G$. Hence, there exists an open neighbourhood $U$ of $x_{0}$ such that $\pi_{n}(i) \pi_{n}\left(U, x_{0}\right) \subseteq q^{-1}(V)$, where $i: U \rightarrow X$ is the inclusion map. Since $H g \cap q^{-1}(V)=\emptyset$, and $\pi_{n}(i) \pi_{n}\left(U, x_{0}\right) \subseteq q^{-1}(V)$, we can conclude that $H g \cap \pi_{n}(i) \pi_{n}\left(U, x_{0}\right)=\emptyset$. Accordingly, for each $g \in G-H$, we can find an open neighbourhood $U$ of $x_{0}$ such that $\pi_{n}(i) \pi_{n}\left(U, x_{0}\right) \cap H g=\emptyset$. Therefore, $X$ is $n$-homotopically Hausdorff relative to $(G, H)$ at $x_{0}$.

Fisher et al. [11, Lemma 2.10] proved that $X$ is homotopically Hausdorff at any point if and only if path space $\widetilde{X}$, containing $\pi_{1}^{w h}\left(X, x_{0}\right)$ as a subspace, is Hausdorff. Therefore, if $X$ is homotopically Hausdorff at any point, then $\pi_{1}^{w h}\left(X, x_{0}\right)$ is Hausdorff. The following corollary shows that the necessary and sufficient condition for $\pi_{n}^{w h}\left(X, x_{0}\right)$ to be Hausdorff is $n$-homotopically Hausdorffness of $X$ at $x_{0}$ for $n \geq 1$. Here, we give a special consequnce of Theorem 3.3, when $G=\pi_{n}\left(X, x_{0}\right)$ and $H=\{e\}$.

Corollary 3.4. Let $\left(X, x_{0}\right)$ be a pointed space, and $n$ be a natural number. Then $X$ is $n$-homotopically Hausdorff at $x_{0}$ if and only if $\pi_{n}^{w h}\left(X, x_{0}\right)$ is Hausdorff.

The whisker topology on homotopy groups depends on the choice of the base point, and the structure of $\pi_{n}^{w h}\left(X, x_{0}\right)$ may differ even in a path component. Brodskiy et al. [6, Corollary 4.9] introduced some spaces, called small loop transfer spaces, on which the topological structure of $\pi_{1}^{w h}\left(X, x_{0}\right)$ homeomorphically transfers by all paths. Pashaei et al. [19] generalized small loop transfer path (SLT path for abbreviation), which was introduced in [6, Definition 4.7]. In the following, we intend to extend this notion to higher dimensions. For this purpose, we need to recall the isomorphism $\Gamma_{\gamma}: \pi_{n}\left(X, x_{0}\right) \rightarrow$ $\pi_{n}\left(X, x_{1}\right)$ induced by a path $\gamma$ from $x_{0}$ to $x_{1}$. See [21, Page 381]. 
Definition 3.5. Let $\gamma$ be a path from $x_{0}$ to $x_{1}$ in $X$. Then for any $n$-loop $\alpha$ at $x_{0}, \gamma_{\#}(\alpha)$ is defined to be an $n$-loop at $x_{1}$, where $\beta:\left(\mathbb{I}^{n}, \mathbb{I}^{n}\right) \rightarrow\left(X, x_{1}\right)$ has the rule $\beta=\beta^{\prime} \circ r$, in which $\beta^{\prime}:\left(\mathbb{I}^{n} \times\{0\}\right) \cup\left(\mathbb{I}^{n} \times \mathbb{I}\right) \rightarrow X$ is defined by $\beta^{\prime}(u, 0)=\alpha(u)$ if $u \in \mathbb{I}^{n}$, and $\beta^{\prime}(u, t)=\gamma(t)$ if $u \in \mathbb{I}^{n}$ and $t \in \mathbb{I}$, and $r: \mathbb{I}^{n} \times \mathbb{I} \rightarrow\left(\mathbb{I}^{n} \times\{0\}\right) \cup\left(\mathbb{I}^{n} \times \mathbb{I}\right)$ is the stereographic retraction.

Theorem 3.6 ([21]). Let $X$ be a space, and let $x_{0}, x_{1} \in X$. For any path $\gamma$ from $x_{0}$ to $x_{1}$, there exists an isomorphism of groups $\Gamma_{\gamma}: \pi_{n}\left(X, x_{0}\right) \rightarrow \pi_{n}\left(X, x_{1}\right)$ defined by $\Gamma_{\gamma}([\alpha])=$ $\left[\gamma_{\#}(\alpha)\right]$.

The isomorphism $\Gamma_{\gamma}: \pi_{n}^{w h}\left(X, x_{0}\right) \rightarrow \pi_{n}^{w h}\left(X, x_{1}\right)$ is not necessarily continuous, but for some paths called SLT paths, $\Gamma_{\gamma}$ is continuous (see [6, Lemma 4.6]). Now, we generalize SLT path to $n$-SLT path for $n \geq 1$, in order to make $\Gamma_{\gamma}$ continuous.

Definition 3.7. Let $X$ be a space, $x_{0}, x_{1} \in X$, and $n \geq 1$. A path $\gamma$ from $x_{0}$ to $x_{1}$ is called small $n$-loop transfer (abbreviated to $n$-SLT), if for every open neighbourhood $U$ of $x_{0}$, there exists an open neighbourhood $V$ of $x_{1}$ such that for every $n$-loop $\beta:\left(\mathbb{I}^{n}, \mathbb{I}^{n}\right) \rightarrow$ $\left(V, x_{1}\right)$, there is an $n$-loop $\alpha:\left(\mathbb{I}^{n}, \mathbb{I}^{n}\right) \rightarrow\left(U, x_{0}\right)$ which is homotopic to $\gamma_{\#}^{-1}(\beta)$.

Brodskiy et al. [6, Lemma 4.6] proved that $\gamma_{\#}: \pi_{1}^{w h}\left(X, x_{0}\right) \rightarrow \pi_{1}^{w h}\left(X, x_{1}\right)$ is continuous if and only if $\gamma^{-1}$ is a 1-SLT path from $x_{0}$ to $x_{1}$. The analogous assertion holds for $n \geq 2$ as follows.

Proposition 3.8. Let $\gamma$ be a path in $X$ from $x_{0}$ to $x_{1}$. Then $\Gamma_{\gamma}: \pi_{n}^{w h}\left(X, x_{0}\right) \rightarrow \pi_{n}^{w h}\left(X, x_{1}\right)$ is continuous if and only if $\gamma^{-1}$ is an n-SLT path.

Proof. By Theorem 3.6 $\Gamma_{\gamma}: \pi_{n}^{w h}\left(X, x_{0}\right) \rightarrow \pi_{n}^{w h}\left(X, x_{1}\right)$ is an isomorphism of groups. Since the whisker topology on the $n$th homotopy group makes it a left topological group, continuity of homomorphisms is equivalent to continuity at the identity [2, Proposition 1.3.4, Page 19]. Thus $\Gamma_{\gamma}$ is continuous if and only if it is continuous at the identity. By definition of the whisker topology, the set $\left\{\pi_{n}\left(i_{2}\right) \pi_{n}\left(V, x_{1}\right) \mid V\right.$ is an open neighbourhood of $\left.x_{1}\right\}$ is a local basis at the identity of $\pi_{n}^{w h}\left(X, x_{1}\right)$. Thus, $\Gamma$ is continuous at the identity if and only if for any open neighbourhood $V$ of $x_{1}, \Gamma_{\gamma}^{-1}\left(\pi_{n}\left(i_{2}\right) \pi_{n}\left(V, x_{0}\right)\right)$ is open in $\pi_{n}^{w h}\left(X, x_{0}\right)$. Again, since $\left\{\pi_{n}\left(i_{1}\right) \pi_{n}\left(U, x_{0}\right) \mid U\right.$ is an open neighbourhood of $\left.x_{0}\right\}$ is a local basis at the identity of $\pi_{n}^{w h}\left(X, x_{0}\right), \Gamma_{\gamma}$ is continuous at the identity if and only if for any open neighbourhood $V$ of $x_{1}$, there is an open neighbourhood $U$ of $x_{0}$ such that $\Gamma_{\gamma}\left(\pi_{n}\left(i_{1}\right) \pi_{n}\left(U, x_{0}\right)\right) \subseteq\left(\pi_{n}\left(i_{2}\right) \pi_{n}\left(V, x_{0}\right)\right)$. That is for any $n$-loop $\alpha$ in $U$ at $x_{0}$, there is an $n$-loop $\beta$ in $V$ at $x_{1}$, such that $\Gamma_{\gamma}([\alpha])=[\beta]$. Since $\Gamma_{\gamma}([\alpha])=\left[\gamma_{\#}(\alpha)\right], \beta$ is homotopic to $\gamma_{\#}(\alpha)$. Equivalently, since $\gamma_{\#}(\alpha) \simeq \gamma_{\#}^{-1-1}(\alpha), \beta$ is homotopic to $\gamma_{\#}^{-1}-1(\alpha)$. Therefore, $\Gamma_{\gamma}$ is continuous if and only if for any open neighbourhood $V$ of $x_{1}$, there is an open neighbourhood $U$ of $x_{0}$, such that for every $n$-loop $\alpha$ in $U$ at $x_{0}$, there is an $n$-loop $\beta$ in $V$ at $x_{1}$ homotopic to $\gamma_{\#}^{-1-1}(\alpha)$, or equivalently, $\gamma^{-1}$ is an $n$-SLT path from $x_{1}$ to $x_{0}$.

Proposition 3.8 implies the following corollary.

Corollary 3.9. Let $X$ be a space, $x_{0}, x_{1} \in X$, and $n \geq 2$. If there is a path $\gamma$ from $x_{0}$ to $x_{1}$ such that $\gamma$ and $\gamma^{-1}$ are $n$-SLT paths, then $\pi_{n}^{w h}\left(X, x_{0}\right)$ and $\pi_{n}^{w h}\left(X, x_{1}\right)$ are isomorphic as topological groups.

\section{Relationship between $L_{n}\left(X, x_{0}\right)$ and $\pi_{n}^{w h}\left(X, x_{0}\right)$}

Let $\varphi: \mathcal{H}_{n}\left(X, x_{0}\right) \rightarrow \prod_{\aleph_{0}} \pi_{n}\left(X, x_{0}\right)$ be the homomorphism (I). In this section, we study the homomorphic image of the Hawaiian group, by the homomorphism $\varphi$, and its relation to the whisker topology on homotopy groups.

It is shown that $L_{n}\left(X, x_{0}\right)$, introduced in [3, Definition 2.6], is equal to $\operatorname{Im}(\varphi)$ and hence it is a subgroup of $\prod_{\aleph_{0}} \pi_{n}\left(X, x_{0}\right)$, for each pointed space $\left(X, x_{0}\right)$. Note that the structure of $L_{n}\left(X, x_{0}\right)$ does not depend only on $\pi_{n}\left(X, x_{0}\right)$. In the following example, for 
every $n \geq 1$ we present two pointed spaces $\left(X, x_{0}\right)$ and $\left(Y, y_{0}\right)$ with $\pi_{n}\left(X, x_{0}\right) \cong \pi_{n}\left(Y, y_{0}\right)$, but $L_{n}\left(X, x_{0}\right) \nRightarrow L_{n}\left(Y, y_{0}\right)$.

Example 4.1. Let $n \geq 1$. Put $Y$ the Eilenberg-MacLane space with $\pi_{n}\left(Y, y_{0}\right) \cong \prod_{\aleph_{0}} \mathbb{Z}$ and $X=\prod_{\aleph_{0}} \mathbb{S}^{n}$. If $x_{0} \in X$, then

$$
\pi_{n}\left(X, x_{0}\right) \cong \prod_{\aleph_{0}} \pi_{n}\left(\mathbb{S}^{n}, 1\right) \cong \prod_{\aleph_{0}} \mathbb{Z} \cong \pi_{n}\left(Y, y_{0}\right)
$$

Since $Y$ is locally $n$-simply connected at $y_{0}, \mathcal{H}_{n}\left(Y, y_{0}\right) \cong L_{n}\left(Y, y_{0}\right) \cong \prod_{\aleph_{0}}^{W} \pi_{n}\left(Y, y_{0}\right)$ (see $\left[17\right.$, Theorem 1]), and therefore, $L_{n}\left(Y, y_{0}\right) \cong \prod_{\aleph_{0}}^{W} \prod_{\aleph_{0}} \mathbb{Z}$.

By a straightforward argument, one can prove that $L_{n}$ preserves the products, for all $n \geq 1$. Thus, $L_{n}\left(X, x_{0}\right) \cong \prod_{\aleph_{0}} L_{n}\left(\mathbb{S}^{n}, 1\right)$. Since $\mathbb{S}^{n}$ is locally $n$-simply connected at 1 , $\mathcal{H}_{n}\left(\mathbb{S}^{n}, 1\right) \cong L_{n}\left(\mathbb{S}^{n}, 1\right) \cong \prod_{\aleph_{0}}^{W} \pi_{n}\left(\mathbb{S}^{n}, 1\right)$ (see [17, Theorem 1]). Therefore, $L_{n}\left(X, x_{0}\right) \cong$ $\prod_{\aleph_{0}} \prod_{\aleph_{0}}^{W} \mathbb{Z}$

Note that $\prod_{\aleph_{0}} \prod_{\aleph_{0}}^{W} \mathbb{Z} ¥ \prod_{\aleph_{0}}^{W} \prod_{\aleph_{0}} \mathbb{Z}$ (see [22]), and hence, $L_{n}\left(X, x_{0}\right) \neq L_{n}\left(Y, y_{0}\right)$.

Example 4.1 shows that the algebraic structure of $\pi_{n}\left(X, x_{0}\right)$ does not determine the structure of $L_{n}\left(X, x_{0}\right)$. But in Theorem 4.7, we will see that the whisker topology on $\pi_{n}\left(X, x_{0}\right)$ can exactly characterize $L_{n}\left(X, x_{0}\right)$. The following theorem manifests the relation between $L_{n}\left(X, x_{0}\right)$ and $\pi_{n}^{w h}\left(X, x_{0}\right)$.

Theorem 4.2. Let $\left(X, x_{0}\right)$ be a pointed space and $n \geq 1$. Then $L_{n}\left(X, x_{0}\right)$ is equal to the set of all sequences converging to the identity in $\pi_{n}^{w h}\left(X, x_{0}\right)$.

Proof. A sequence $\left\{\left[\alpha_{k}\right]\right\}_{\aleph_{0}}$ belongs to $L_{n}\left(X, x_{0}\right)$ if and only if there exists null-convergent sequence $\left\{\beta_{k}\right\}_{\aleph_{0}}$ with $\alpha_{k} \simeq \beta_{k}$ for every $k \in \mathbb{N}$. A sequence $\left\{\beta_{k}\right\}_{\aleph_{0}}$ is null-convergent if and only if for each open set $U$ of $x_{0}$ there exists $K \in \mathbb{N}$ such that if $k \geq K$, then $i m\left(\beta_{k}\right) \subseteq U$. Recall that $i m\left(\beta_{k}\right) \subseteq U$ if and only if there exists $\gamma:\left(\mathbb{S}^{n}, 1\right) \rightarrow\left(U, x_{0}\right)$ such that $\beta_{k} \simeq i \circ \gamma$, where $i: U \rightarrow X$ is the inclusion map. Hence, $\left\{\beta_{k}\right\}_{\aleph_{0}}$ is null-convergent if and only if there exists $K \in \mathbb{N}$ such that if $k \geq K$, then $\left[\beta_{k}\right] \in\left\{[i \circ \gamma] \mid \gamma\right.$ is an $n$-loop at $x_{0}$ in $U\}=\pi_{n}(i) \pi_{n}\left(U, x_{0}\right)$, or equivalently $\left[\alpha_{k}\right] \in \pi_{n}(i) \pi_{n}\left(U, x_{0}\right)$.

Therefore, $\left\{\left[\alpha_{k}\right]\right\}_{\aleph_{0}} \in L_{n}\left(X, x_{0}\right)$ if and only if for each open set $U$ of $x_{0}$, there exists $K \in \mathbb{N}$ such that if $k \geq K$, then $\left[\alpha_{k}\right] \in \pi_{n}(i) \pi_{n}\left(U, x_{0}\right)$. Since the set $\left\{\pi_{n}(i) \pi_{n}\left(U, x_{0}\right) \mid U\right.$ is an open subset of $\left.x_{0}\right\}$ forms a local basis for the whisker topology on $\pi_{n}\left(X, x_{0}\right)$ at the identity, $\left\{\left[\alpha_{k}\right]\right\}_{\aleph_{0}} \in L_{n}\left(X, x_{0}\right)$ if and only if $\left\{\left[\alpha_{k}\right]\right\}_{\aleph_{0}}$ converges to the identity in $\pi_{n}^{w h}\left(X, x_{0}\right)$.

Recall that by the definition of whisker topology on the $n$th homotopy group of pointed space $\left(X, x_{0}\right), \pi_{n}^{w h}\left(X, x_{0}\right)$ is indiscrete if and only if all $n$-loops in $X$ at $x_{0}$ are small. Also by Proposition $3.1 \pi_{n}^{w h}\left(X, x_{0}\right)$ is discrete if and only if $X$ is semi-locally $n$-simply connected at $x_{0}$.

Corollary 4.3. Let $X$ be a space having a countable local basis at $x_{0}$.

(1) $X$ is semi-locally $n$-simply connected at $x_{0}$ if and only if $L_{n}\left(X, x_{0}\right)=\prod_{\aleph_{0}}^{W} \pi_{n}\left(X, x_{0}\right)$.

(2) All n-loops at $x_{0}$ are small if and only if $L_{n}\left(X, x_{0}\right)=\prod_{\aleph_{0}} \pi_{n}\left(X, x_{0}\right)$.

Proof. Since $X$ has a countable local basis at $x_{0}, \pi_{n}^{w h}\left(X, x_{0}\right)$ is first countable, by Lemma 2.3 .

(1) $\pi_{n}^{w h}\left(X, x_{0}\right)$ is discrete if and only if every convergent sequence is eventually constant. Since $\pi_{n}^{w h}\left(X, x_{0}\right)$ is a left topological group, every convergent sequence is obtained by some left translation from a sequence converging to the identity. Hence by Theorem 4.2 the result holds.

(2) If $\pi_{n}^{w h}\left(X, x_{0}\right)$ is indiscrete, then all sequences are convergent. Hence, all sequences in $\pi_{n}^{w h}\left(X, x_{0}\right)$ converge to the identity. By Theorem $4.2, L_{n}\left(X, x_{0}\right)$ equals the 
set of convergent sequences to the identity of $\pi_{n}^{w h}\left(X, x_{0}\right)$, and then $L_{n}\left(X, x_{0}\right)=$ $\prod_{\aleph_{0}} \pi_{n}\left(X, x_{0}\right)$.

Conversely, if $L_{n}\left(X, x_{0}\right)=\prod_{\aleph_{0}} \pi_{n}\left(X, x_{0}\right)$, then all sequences converge to the identity in $\pi_{n}^{w h}\left(X, x_{0}\right)$. It is equivalent to $\pi_{n}^{w h}\left(X, x_{0}\right)$ be indiscrete at the identity. Since $\pi_{n}^{w h}\left(X, x_{0}\right)$ is a left topological group, it is indiscrete at every point.

Note that the $n$-Hawaiian earring space, $\mathbb{H} \mathbb{E}^{n}$, does not belong to the two classes of Corollary 4.3, and hence $\pi_{n}^{w h}\left(\mathbb{H}^{n}, \theta_{0}\right)$ is not discrete nor indiscrete. The $n$-Hawaiian earring space was generalized to $n$-Hawaiian like spaces by Ghane et al. [14] as a specified topology on disjoint union of CW spaces with a common point as follows.

Definition 4.4 ([14]). Let $\left\{X_{i}\right\}_{i \in \mathbb{N}}$ be a family of topological spaces. Suppose that the underlying set of $\widetilde{\bigvee}_{i \in \mathbb{N}} X_{i}$ is the disjoint union of $X_{i}$ 's with exactly one point $x_{*}$ in common, equipped with a topology generated by the neighbourhood bases as follows.

(1) If $x \in X_{i} \backslash\left\{x_{*}\right\}$, then the neighbourhood basis of $\widetilde{\bigvee}_{i \in \mathbb{N}} X_{i}$ at $x$ is the one of $X_{i}$, $i \in \mathbb{N}$.

(2) At point $x_{*}$, the neighbourhood basis consists of sets of the form $\bigcup_{i \in \mathbb{N} \backslash F} X_{i} \cup$ $\bigcup_{i \in F} U_{i}$, where $F$ is a finite set of natural numbers and $U_{i}$ is an open neighbourhood of $x_{*}$ in $X_{i}$.

The space $\widetilde{\mathrm{V}}_{i \in \mathbb{N}} X_{i}$ is called an $n$-Hawaiian like space, when $X_{i}$ 's are all $(n-1)$-connected compact CW spaces.

Let $\pi_{n}^{q t o p}\left(X, x_{*}\right)$ denote the quasi-topological $n$th homotopy group induced by the compactopen topology on the $n$-loop space $\Omega_{n}\left(X, x_{*}\right)$ (see [13]). If $X=\widetilde{V}_{i \in \mathbb{N}} X_{i}$ is an $n$-Hawaiian like space, then for $n \geq 2$, it was shown in [14, Theorem 1.1] that $\pi_{n}\left(X, x_{*}\right) \cong \prod_{i \in \mathbb{N}} \pi_{n}\left(X_{i}, x_{*}\right)$ and that $\pi_{n}^{q t o p}\left(X, x_{*}\right)$ is isomorphic to the prodiscrete topological group $\prod_{i \in \mathbb{N}} \pi_{n}\left(X_{i}, x_{*}\right)$. In [14, Theorem 3.3], Ghane et al. proved that the topologies of $\pi_{n}^{\lim }\left(X, x_{*}\right)$ and $\pi_{n}^{q t o p}\left(X, x_{*}\right)$ coincide if $X$ is an $n$-Hawaiian like space.

Let $\left\{X_{i}\right\}_{i \in \mathbb{N}}$ be a family of spaces each of which is Tychonoff, $(n-1)$-connected, locally strongly contractible and first countable at $x_{i}$. We call $X=\widetilde{V}_{i \in \mathbb{N}} X_{i}$, the compact union of the above family, the generalized $n$-Hawaiian like space. In [9, Theorem 1.1], it was proved that for $n \geq 2, \pi_{n}\left(X, x_{*}\right) \cong \prod_{\mathbb{N}} \pi_{n}\left(X_{i}, x_{*}\right)$. In the following proposition, we show that for generalized $n$-Hawaiian like spaces, the topology of $\pi_{n}^{w h}\left(X, x_{*}\right)$ is prodiscrete.

Proposition 4.5. If $X=\bigvee_{i \in \mathbb{N}} X_{i}$ is a generalized $n$-Hawaiian like space and $x_{*}$ is the common point, $n \geq 2$, then $\pi_{n}^{w h}\left(X, x_{*}\right)$ is isomorphic to the prodiscrete topological group $\prod_{i \in \mathbb{N}} \pi_{n}\left(X_{i}, x_{*}\right)$.

Proof. Using the isomorphism $\pi_{n}\left(X, x_{*}\right) \cong \prod_{\mathbb{N}} \pi_{n}\left(X_{i}, x_{*}\right)$, we can consider elements of $\pi_{n}\left(X, x_{*}\right)$ by the corresponding ones of $\prod_{i \in \mathbb{N}} \pi_{n}\left(X_{i}, x_{*}\right)$. That is $[f] \in \pi_{n}\left(X, x_{*}\right)$ can be considered as $\left(\left[f^{1}\right],\left[f^{2}\right], \ldots\right) \in \prod_{i \in \mathbb{N}} \pi_{n}\left(X_{i}, x_{*}\right)$, where $f^{i}=r_{i} \circ f$ and $r_{i}: X \rightarrow X_{i}$ is the natural retraction. Since $X$ is first countable and $n$-homotopically Hausdorff at $x_{*}$, then $\pi_{n}^{w h}\left(X, x_{*}\right)$ is a metric topological group by Corollary 2.7. Thus, the topology of $\pi_{n}^{w h}\left(X, x_{*}\right)$ is identified by convergent sequences. By Theorem 4.2 , the set of convergent sequences to the identity of $\pi_{n}^{w h}\left(X, x_{*}\right)$ is equal to $L_{n}\left(X, x_{*}\right)$. It suffices to verify that $\left\{\left(\left[f_{k}^{1}\right],\left[f_{k}^{2}\right], \ldots\right)\right\}_{k \in \mathbb{N}} \in L_{n}\left(X, x_{*}\right)$ if and only if it converges to the identity in prodiscrete topological group $\prod_{i \in \mathbb{N}} \pi_{n}\left(X_{i}, x_{*}\right)$. Let $\left\{\left(\left[f_{k}^{1}\right],\left[f_{k}^{2}\right], \ldots\right)\right\}_{\mathbb{N}} \in L_{n}\left(X, x_{*}\right)$. We must show that for any open set $U$ of the identity in $\prod_{i \in \mathbb{N}} \pi_{n}\left(X_{i}, x_{*}\right),\left(\left[f_{k}^{1}\right],\left[f_{k}^{2}\right], \ldots\right) \in U$ for all $k \in \mathbb{N}$ except a finite number. The elements of the local basis at the identity of $\prod_{i \in \mathbb{N}} \pi_{n}\left(X_{i}, x_{*}\right)$ are of the form $U_{i}=\left\{e_{1}\right\} \times\left\{e_{2}\right\} \times \cdots \times\left\{e_{i-1}\right\} \times \pi_{n}\left(X_{i}, x_{*}\right) \times \pi_{n}\left(X_{i+1}, x_{*}\right) \times \cdots$, for some $i \in \mathbb{N}$, where $e_{1}, e_{2}, \ldots$ are the identity elements of $\pi_{n}\left(X_{1}, x_{*}\right), \pi_{n}\left(X_{2}, x_{*}\right), \ldots$, respectively. By [3, Proof of Theorem 2.10], $\left\{\left(\left[f_{k}^{1}\right],\left[f_{k}^{2}\right], \ldots\right)\right\}_{k \in \mathbb{N}} \in L_{n}\left(X, x_{*}\right)$ if and only if $\left[f_{k}^{j}\right]$ is the 
identity element for all $j \in \mathbb{N}$ except a finite number. Thus, for every $j \in \mathbb{N}$, there exists $K_{j} \in \mathbb{N}$ such that if $k \geq K_{j}$, then $\left[f_{k}^{j}\right]=e_{j}$. Put $K=\max \left\{K_{1}, K_{2}, \ldots, K_{i-1}\right\}$. If $k \geq K$, then $\left[f_{k}^{j}\right]=e_{j}$, for $j<i$. Therefore, $\left(\left[f_{k}^{1}\right],\left[f_{k}^{2}\right], \ldots\right) \in U_{i}$ if $k \geq K$. That is $\left\{\left(\left[f_{k}^{1}\right],\left[f_{k}^{2}\right], \ldots\right)\right\}_{k \in \mathbb{N}}$ converges to the identity in $\prod_{i \in \mathbb{N}} \pi_{n}\left(X_{i}, x_{*}\right)$.

Conversely, let $\left\{\left(\left[f_{k}^{1}\right],\left[f_{k}^{2}\right], \ldots\right)\right\}_{k \in \mathbb{N}}$ converges to the identity in $\prod_{i \in \mathbb{N}} \pi_{n}\left(X_{i}, x_{*}\right)$. By the form of the local basis at the identity in $\prod_{i \in \mathbb{N}} \pi_{n}\left(X_{i}, x_{*}\right), U_{i}$ 's, there exists $K_{i+1} \in \mathbb{N}$ such that if $k \geq K_{i+1}$, then $\left[f_{k}^{j}\right]=e_{j}$ for $j \leq i$. Equivalently, $\left[f_{k}^{i}\right]$ 's are identity element except possibly finite numbers $k<K_{i+1}$. Again, by [3, Proof of Theorem 2.10], the sequence $\left\{\left(\left[f_{k}^{1}\right],\left[f_{k}^{2}\right], \ldots\right)\right\}_{k \in \mathbb{N}}$ belongs to $L_{n}\left(X, x_{*}\right)$.

Example 4.6. For the $n$-dimensional Hawaiian earring, $\mathbb{H} \mathbb{E}^{n}$, it is proved that $\pi_{n}\left(\mathbb{H} \mathbb{E}^{n}\right) \cong$ $\prod_{\mathbb{N}} \mathbb{Z}\left[9\right.$, Corollary 1.2]. Then $\pi_{n}^{w h}\left(\mathbb{H}^{n}, \theta\right)$ is isomorphic to the prodiscrete topological group of $\prod_{\mathbb{N}} \mathbb{Z}$.

Theorem 4.2 shows that there exists a close relation between $L_{n}\left(X, x_{0}\right)$ and $\pi_{n}^{w h}\left(X, x_{0}\right)$. In the following theorem, we prove that the structure of $\pi_{n}^{w h}\left(X, x_{0}\right)$ fixes the structure of $L_{n}\left(X, x_{0}\right)$. Note that an isomorphism of left topological groups is an isomorphism of groups which is also a homeomorphism on the underlying topological space.

Theorem 4.7. Let $\left(X, x_{0}\right)$ and $\left(Y, y_{0}\right)$ be two pointed spaces and let $n \geq 1$. If $\pi_{n}^{w h}\left(X, x_{0}\right) \cong$ $\pi_{n}^{w h}\left(Y, y_{0}\right)$ as left topological groups, then $L_{n}\left(X, x_{0}\right) \cong L_{n}\left(Y, y_{0}\right)$. Moreover, if $X$ and $Y$ have countable local bases at $x_{0}$ and $y_{0}$, respectively, and if the isomorphism $L_{n}\left(X, x_{0}\right) \cong$ $L_{n}\left(Y, y_{0}\right)$ is induced by some isomorphism $g: \pi_{n}\left(X, x_{0}\right) \rightarrow \pi_{n}\left(Y, y_{0}\right)$, then $g$ is a homeomorphism.

Proof. Let $g: \pi_{n}^{w h}\left(X, x_{0}\right) \rightarrow \pi_{n}^{w h}\left(Y, y_{0}\right)$ be an isomorphism of left topological groups. Since $g$ is an isomorphism from $\pi_{n}\left(X, x_{0}\right)$ onto $\pi_{n}\left(Y, y_{0}\right)$, it induces monomorphisms $\widetilde{g}$ : $L_{n}\left(X, x_{0}\right) \rightarrow \prod_{\aleph_{0}} \pi_{n}\left(Y, y_{0}\right)$ and $\widetilde{g^{-1}}: L_{n}\left(Y, y_{0}\right) \rightarrow \prod_{\aleph_{0}} \pi_{n}\left(X, x_{0}\right)$ by the rule $\widetilde{g}\left(\left\{\left[\alpha_{k}\right]\right\}_{\aleph_{0}}\right)=$ $\left\{g\left(\left[\alpha_{k}\right]\right)\right\}_{\aleph_{0}}$ and $\widetilde{g^{-1}}\left(\left\{\left[\beta_{k}\right]\right\}_{\aleph_{0}}\right)=\left\{g^{-1}\left(\left[\beta_{k}\right]\right)\right\}_{\aleph_{0}}$, respectively. We show that $\widetilde{g}\left(L_{n}\left(X, x_{0}\right)\right) \subseteq$ $L_{n}\left(Y, y_{0}\right)$ and $\widetilde{g^{-1}}\left(L_{n}\left(Y, y_{0}\right)\right) \subseteq L_{n}\left(X, x_{0}\right)$. Let $\left\{\left[\alpha_{k}\right]\right\}_{\aleph_{0}} \in L_{n}\left(X, x_{0}\right)$, then by Theorem 4.2, $\left\{\left[\alpha_{k}\right]\right\}_{\aleph_{0}}$ converges to the identity in $\pi_{n}^{w h}\left(X, x_{0}\right)$. Since $g$ is a continuous homomorphism, $\left\{g\left(\left[\alpha_{k}\right]\right)\right\}_{\aleph_{0}}$ converges to the identity in $\pi_{n}^{w h}\left(Y, y_{0}\right)$. By Theorem 4.2, $\widetilde{g}\left(\left\{\left[\alpha_{k}\right]\right\}_{\aleph_{0}}\right)=\left\{g\left(\left[\alpha_{k}\right]\right)\right\}_{\aleph_{0}} \in L_{n}\left(Y, y_{0}\right)$. Since $\left\{\left[\alpha_{k}\right]\right\}_{\aleph_{0}}$ is an arbitrary element of $L_{n}\left(X, x_{0}\right)$, it implies that $\widetilde{g}\left(L_{n}\left(X, x_{0}\right)\right) \subseteq L_{n}\left(Y, y_{0}\right)$. A similar argument can be applied to show that $\widetilde{g^{-1}}\left(L_{n}\left(Y, y_{0}\right)\right) \subseteq L_{n}\left(X, x_{0}\right)$. Moreover,

$$
\widetilde{g} \circ \widetilde{g^{-1}}\left(\left\{\left[\beta_{k}\right]\right\}_{\aleph_{0}}\right)=\widetilde{g}\left(\left\{g^{-1}\left[\beta_{k}\right]\right\}_{\aleph_{0}}\right)=\left\{g \circ g^{-1}\left[\beta_{k}\right]\right\}_{\aleph_{0}}=\left\{\left[\beta_{k}\right]\right\}_{\aleph_{0}} .
$$

Hence $\widetilde{g} \circ \widetilde{g^{-1}}=i d_{L_{n}\left(Y, y_{0}\right)}$. Similarly $\widetilde{g^{-1}} \circ \widetilde{g}=i d_{L_{n}\left(X, x_{0}\right)}$. Therefore $\widetilde{g}: L_{n}\left(X, x_{0}\right) \cong$ $L_{n}\left(Y, y_{0}\right)$.

Conversely, let $g: \pi_{n}\left(X, x_{0}\right) \rightarrow \pi_{n}\left(Y, y_{0}\right)$ be the isomorphism inducing $h: L_{n}\left(X, x_{0}\right) \cong$ $L_{n}\left(Y, y_{0}\right)$ by the rule $h\left(\left\{\left[\alpha_{k}\right]\right\}_{\aleph_{0}}\right)=\left\{g\left(\left[\alpha_{k}\right]\right)\right\}_{\aleph_{0}}$. We must show that $g$ and $g^{-1}$ are continuous. Since $g$ and $g^{-1}$ are homomorphisms and also $\pi_{n}^{w h}\left(X, x_{0}\right)$ and $\pi_{n}^{w h}\left(Y, y_{0}\right)$ are left topological groups, $g$ and $g^{-1}$ are continuous if they are continuous at the identities by [2, Proposition 1.3.4]. Moreover, since $X$ and $Y$ are first countable at $x_{0}$ and $y_{0}$, respectively, $\pi_{n}^{w h}\left(X, x_{0}\right)$ and $\pi_{n}^{w h}\left(Y, y_{0}\right)$ are first countable spaces. Thus, to prove continuity of $g$ and $g^{-1}$, it suffices to check sequential continuity at the identities. Let $\left\{\left[\alpha_{k}\right]\right\}_{\aleph_{0}}$ be a sequence converges to the identity in $\pi_{n}^{w h}\left(X, x_{0}\right)$. By Theorem 4.2, $\left\{\left[\alpha_{k}\right]\right\}_{\aleph_{0}} \in L_{n}\left(X, x_{0}\right)$. Since $h\left(L_{n}\left(X, x_{0}\right)\right) \subseteq L_{n}\left(Y, y_{0}\right), h$ maps $\left\{\left[\alpha_{k}\right]\right\}_{\aleph_{0}}$ into $L_{n}\left(Y, y_{0}\right)$. Again by Theorem $4.2, h\left(\left\{\left[\alpha_{k}\right]\right\}_{\aleph_{0}}\right)$ converges to the identity in $\pi_{n}^{w h}\left(Y, y_{0}\right)$. Moreover, $h\left(\left\{\left[\alpha_{k}\right]\right\}_{\aleph_{0}}\right)=\left\{g\left(\left[\alpha_{k}\right]\right)\right\}_{\aleph_{0}}$. Therefore, $\left\{g\left(\left[\alpha_{k}\right]\right)\right\}_{\aleph_{0}}$ converges to the identity. Since $g$ is a homomorphism, $g$ maps the identity of $\pi_{n}^{w h}\left(X, x_{0}\right)$ to the identity element of $\pi_{n}^{w h}\left(Y, y_{0}\right)$. Thus, $g$ is sequentially continuous at the identity as required. Similarly, by using the 
inclusion $h^{-1}\left(L_{n}\left(Y, y_{0}\right)\right) \subseteq L_{n}\left(X, x_{0}\right)$, one can show that $g^{-1}$ is continuous. Thus, $g$ and $g^{-1}$ are continuous maps, and hence $g$ is a homeomorphism.

Let $x_{0}, x_{1} \in X$. If there exists a path $\gamma$ from $x_{0}$ to $x_{1}$, then $\gamma_{\#}$ in Definition 3.5 induces an isomorphism from $\pi_{n}\left(X, x_{0}\right)$ onto $\pi_{n}\left(X, x_{1}\right)$. But there exist path connected spaces, namely $\mathbb{H}^{n}, n \geq 2$, such that $L_{n}\left(\mathbb{H}_{\mathbb{E}}^{n}, \theta\right) \neq L_{n}\left(\mathbb{H} \mathbb{E}^{n}, a\right)$, where $a \neq \theta$ (see [3, Corollary 2.11]). By Theorem 4.7 and Corollary 3.9, $\gamma_{\#}$ can analogously transfer $L_{n}\left(X, x_{0}\right)$ isomorphically onto $L_{n}\left(X, x_{1}\right)$, if $\gamma$ and $\gamma^{-1}$ are $n$-SLT paths.

Corollary 4.8. Let $X$ have countable local bases at two points $x_{0}$ and $x_{1}$, and $n \geq 1$. If there exists a path $\gamma$ from $x_{0}$ to $x_{1}$, such that $\gamma$ and $\gamma^{-1}$ are $n$-SLT paths, then $\left\{\Gamma_{\gamma}\right\}_{\aleph_{0}}$ : $L_{n}\left(X, x_{0}\right) \rightarrow L_{n}\left(X, x_{1}\right)$ is an isomorphism.

By Corollary 4.8, if $\varphi: \mathcal{H}_{n}\left(X, x_{0}\right) \rightarrow L_{n}\left(X, x_{0}\right)$ is injective, and $\gamma$ and $\gamma^{-1}$ are $n$-SLT paths, then $\left\{\Gamma_{\gamma}\right\}_{\aleph_{0}}$ induces an isomorphism from $\mathcal{H}_{n}\left(X, x_{0}\right)$ onto $\mathcal{H}_{n}\left(X, x_{1}\right)$. For instance, on semilocally $n$-simply connected spaces, we have such an isomorphism.

The harmonic archipelago, $\mathbb{H} \mathbb{A}$, is a non-simply connected space with small loops. The fundamental group and homology groups of the harmonic archipelago were studied in [8] and [18], respectively. Here, we recall some of their results to use in Example 4.10.

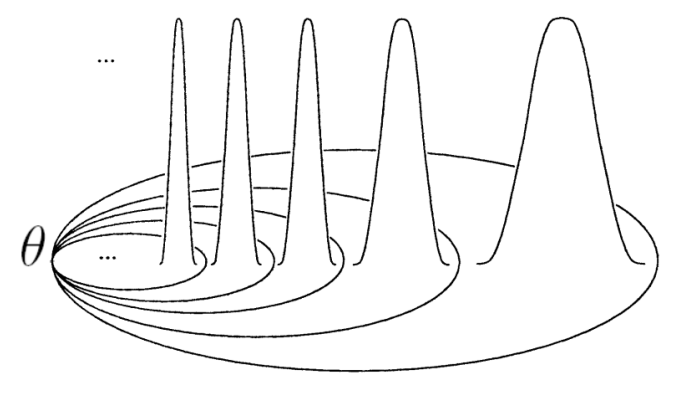

Theorem 4.9 ([8,18]). Let $\times^{\sigma}$ denote the free $\sigma$-product of a family of groups, and $\bar{H}^{N}$ denote the normal closure of the subgroup $H$ in a given group. Then

(i) [8, Theorem 5]

$$
\pi_{1}(\mathbb{H} \mathbb{A}) \cong \frac{\times_{\aleph_{0}}^{\sigma} \mathbb{Z}}{{ }_{\aleph_{0}} \mathbb{Z}} \mathbb{Z}^{N}
$$

(ii) [18, Theorem 1.2 and Proposition 2.4]. Let $P$ be the set of all prime numbers. Then

$$
H_{1}(\mathbb{H} \mathbb{A}) \cong \frac{\prod_{\aleph_{0}} \mathbb{Z}}{\sum_{\aleph_{0}} \mathbb{Z}} \cong\left(\prod_{p \in P} A_{p}\right) \oplus\left(\sum_{c} \mathbb{Q}\right),
$$

where $A_{p}$ is the $p$-adic completion of the direct sum of p-adic integers $\sum_{c} \mathbb{J}_{p}$, and c denotes the continuum cardinal.

Example 4.10 illustrates that Corollary 4.8 does not hold if there is no such path between the points.

Example 4.10. Let $\mathbb{H} \mathbb{A}$ be the harmonic archipelago space, and $\theta$ be the origin.

Let $a \in \mathbb{H} \mathbb{A}$ and $a \neq \theta$. Then by Corollary $4.3, L_{1}(\mathbb{H} \mathbb{A}, a)=\prod_{\aleph_{0}}^{W} \pi_{1}(\mathbb{H} \mathbb{A}, a)$ and $L_{1}(\mathbb{H} \mathbb{A}, \theta)=\prod_{\aleph_{0}} \pi_{1}(\mathbb{H} \mathbb{A}, \theta)$. By Theorem $4.9(\mathrm{i})$ since $\pi_{1}(\mathbb{H} \mathbb{A}) \cong \frac{\times_{\aleph_{0}}^{\sigma} \mathbb{Z}}{{ }_{\aleph_{0}} \mathbb{Z}^{N}}$, we have

$$
L_{1}(\mathbb{H} \mathbb{A}, a) \cong \prod_{\aleph_{0}}^{W} \frac{\times_{\aleph_{0}}^{\sigma} \mathbb{Z}}{{ }_{* \aleph_{0}} \mathbb{Z}^{N}}, \quad L_{1}(\mathbb{H} \mathbb{A}, \theta) \cong \prod_{\aleph_{0}} \frac{\times_{\aleph_{0}}^{\sigma} \mathbb{Z}}{{ }_{\aleph_{0}} \mathbb{Z}^{N}} .
$$

We prove that $L_{1}(\mathbb{H} \mathbb{A}, a) \nRightarrow L_{1}(\mathbb{H} \mathbb{A}, \theta)$. By contrary, assume that $L_{1}(\mathbb{H} \mathbb{A}, a) \cong L_{1}(\mathbb{H} \mathbb{A}, \theta)$. Thus, their abelianizations must be isomorphic. That is $A b\left(\prod_{\aleph_{0}}^{W} \frac{\times_{\aleph_{0}}^{\sigma} \mathbb{Z}}{{ }_{\aleph_{0}} \mathbb{Z}}\right) \cong \sum_{\aleph_{0}} A b\left(\frac{\times_{\aleph_{0}}^{\sigma} \mathbb{Z}}{{ }_{\aleph_{0}} \mathbb{Z}^{N}}\right) \cong$ 
$A b\left(\prod_{\aleph_{0}} \frac{\times_{\aleph_{0}}^{\sigma} \mathbb{Z}}{{ }_{\aleph_{0}} \mathbb{Z}^{N}}\right)$. Let $G=\prod_{\aleph_{0}} \frac{\times_{\aleph_{0}}^{\sigma} \mathbb{Z}}{{ }_{\aleph_{0}} \mathbb{Z}^{N}}$. Then $G$ is the fundamental group of the countably infinite product of copies of the harmonic archipelago. Thus $G$ is the fundamental group of a space $X$ in which each based loop has arbitrarily small representatives. Then by [15, Theorem 4], we know $G$ satisfies the property of being Higman-complete. Moreover, the first singular homology $H_{1}(X)$ is isomorphic to the abelianization of $G$. Since we are assuming that $G$ is isomorphic to the weak direct product $\prod_{\aleph_{0}}^{W} \frac{\times_{\aleph_{0}}^{\sigma} \mathbb{Z}}{\frac{{ }_{\aleph_{0}} \mathbb{Z}}{\mathbb{Z}^{N}}}$ and the abelianization of a weak direct product can be computed coordinatewise, we get that the abelianization of $G$ is isomorphic to $\sum_{\aleph_{0}}\left(\left(\prod_{p \in P} A_{p}\right) \oplus\left(\sum_{c} \mathbb{Q}\right)\right)$. In particular, the abelianization of $G$ is torsion-free. Then by [15, Corollary 5], since $A b(G) \cong H_{1}(X)$ is torsion-free it must be algebraically compact. Now $\sum_{\aleph_{0}}\left(\prod_{p \in P} A_{p}\right)$ is algebraically compact as a direct summand of the algebraically compact abelian group $A b(G)$. Moreover, the group $A_{p}$ is the $p$-adic completion of $\sum_{c} \mathbb{J}_{p}$, and thus it is complete in $p$-adic topology. By [12, p. 163, Remark], since $p$-adic topology is coarser than $\mathbb{Z}$-adic topology, $A_{p}$ is reduced algebraically compact. By [12, p. 101, Exercise 5], a direct sum or a direct product of groups is reduced if and only if every component is reduced. Therefore, $\sum_{\mathbb{N}} \prod_{p \in P} A_{p}$ is reduced algebraically compact. By [12, p. 163, Theorem 19.1], a group is complete in the $\mathbb{Z}$-adic topology if and only if it is reduced algebraically compact. Thus, $\sum_{\mathbb{N}} \prod_{p \in P} A_{p}$ is complete in $\mathbb{Z}$-adic topology. Also, by [12, p. 166, Corollary 39.10] if $A=\sum_{i \in I} C_{i}$ is a direct decomposition of a complete group $A$, then all the $C_{i}$ are complete groups, and there is an integer $n>0$ such that $n C_{i}=0$ for almost all $i \in I$. Hence, there is an integer $n>0$ such that $n \prod_{p \in P} A_{p}=0$. It is equivalent to $\prod_{p \in P} A_{p}$ being torsion group, which is a contradiction. Therefore, there is no isomorphism from $L_{1}(\mathbb{H} \mathbb{A}, a)$ onto $L_{1}(\mathbb{H} \mathbb{A}, \theta)$.

Note that $\pi_{1}^{w h}(\mathbb{H} \mathbb{A}, a)$ is isomorphic to the discrete topological group $\frac{\times_{\aleph_{0}}^{\sigma} \mathbb{Z}}{{ }_{*_{\aleph_{0}} \mathbb{Z}^{N}}^{N}}$, and $\pi_{1}^{w h}(\mathbb{H} \mathbb{A}, \theta)$ is isomorphic to indiscrete one. Hence, there is no isomorphism of left topological groups from $\pi_{1}^{w h}(\mathbb{H} \mathbb{A}, a)$ onto $\pi_{1}^{w h}(\mathbb{H} \mathbb{A}, \theta)$, but one can not deduce that $L_{1}(\mathbb{H} \mathbb{A}, a) \neq L_{1}(\mathbb{H} \mathbb{A}, \theta)$.

Acknowledgment. The authors would like to thank the referees for their careful reading and useful comments and suggestions that helped to improve the article.

\section{References}

[1] M. Abdullahi Rashid, N. Jamali, B. Mashayekhy, S.Z. Pashaei and H. Torabi, On subgroup topologies on fundamental groups, doi:10.15672/hujms.464056.

[2] A. Arhangegel'skii and M. Tkachenko, Topological Groups and Related Structures, Atlantis Press, Amsterdam, 2008.

[3] A. Babaee, B. Mashayekhy and H. Mirebrahimi, On Hawaiian groups of some topological spaces, Topology Appl. 159 (8), 2043-2051, 2012.

[4] W.A. Bogley and A.J. Sieradski, Universal path spaces, http://people. oregonstate.edu/ bogleyw/research/ups.pdf.

[5] N. Brodskiy, J. Dydak, B. Labuz and A. Mitra, Covering maps for locally path connected spaces, Fund. Math. 218, 13-46, 2012.

[6] N. Brodskiy, J. Dydak, B. Labuz and A. Mitra, Topological and uniform structures on universal covering spaces, arXiv:1206.0071, 2012.

[7] G.R. Conner and J. Lamoreaux, On the existence of universal covering spaces for metric spaces and subsets of the Euclidean plane, Fund. Math. 187, 95-110, 2005.

[8] G.R. Conner, W. Hojka and M. Meilstrup, Archipelago groups, Proc. Amer. Math. Soc. 143, 4973-4988, 2015.

[9] K. Eda and K. Kawamura, Homotopy and homology groups of the n-dimensional Hawaiian Earring, Fund. Math. 165 (1), 17-28, 2000. 
[10] P. Fabel, Multiplication is discontinuous in the Hawaiian Earring droup (with the Quotient Topology), Bull. Pol. Acad. Sci. Math. 59 (1), 77-83, 2011.

[11] H. Fischer and A. Zastrow, Generalized universal coverings and the shape group, Fund. Math. 197, 167-196, 2007.

[12] L. Fuchs, Infinite Abelian Groups I, Academic Press, New York, 1970.

[13] F.H. Ghane, Z. Hamed, B. Mashayekhy, and H. Mirebrahimi, Topological homotopy groups, Bull. Belg. Math. Soc. Simon Stevin, 15 (3), 455-464, 2008.

[14] F.H. Ghane, Z. Hamed, B. Mashayekhy and H. Mirebrahimi, On topological homotopy groups of n-Hawaiian like spaces, Topology Proc. 36, 255-266, 2010.

[15] Herfort and Hojka, Cotorsion and wild homology, Israel J. Math. 221, 275-290, 2017.

[16] N. Jamali, B. Mashayekhy, H. Torabi, S.Z. Pashaei and M. Abdullahi Rashid, On topologized fundamental groups with small loop transfer viewpoints, Acta Math. Vietnamica, 43, 1-27, 2018.

[17] U.H. Karimov and D. Repovš, Hawaiian groups of topological spaces (Russian), Uspekhi. Mat. Nauk. 61 (5), 185-186, 2006; transl. in Russian Math. Surv. 61 (5), 987-989, 2006.

[18] U.H. Karimov and D. Repovš, On the homology of the Harmonic archipelago, Central European J. Math. 10, 863-872, 2012.

[19] S.Z. Pashaei, B. Mashayekhy, H. Torabi and M. Abdullahi Rashid, Small loop transfer spaces with respect to subgroups of fundamental groups, Topology Appl. 232, 242-255, 2017.

[20] H. Passandideh, F.H. Ghane and Z. Hamed, On the homotopy groups of separable metric spaces, Topology Appl. 158, 1607-1614, 2011.

[21] E.H. Spanier, Algebraic Topology, McGraw-Hill, New York, 1966.

[22] B. Zimmermann-Huisgen, On Fuchs' problem 76, J. Reine Angew. Math. 309, 86-91, 1979. 